\title{
Treatment modification after starting CART in people living with HIV: retrospective analysis of the German ClinSurv HIV Cohort 2005-2017
}

\author{
Melanie Stecher ${ }^{1,2}\left(\mathbb{D} \cdot\right.$ Philipp Schommers ${ }^{1,2} \cdot$ Christian Kollan $^{3} \cdot$ Matthias Stoll $^{4} \cdot$ Frieder Kuhlendahl $^{5}$. \\ Hans-Jürgen Stellbrink ${ }^{6}$. Jan-Christian Wasmuth ${ }^{7,8} \cdot$ Christoph Stephan $^{9} \cdot$ Laura Hamacher $^{1} \cdot$ Clara Lehmann $^{1,2}$. \\ Christoph Boesecke ${ }^{7}$. Johannes Bogner ${ }^{10}$. Stefan Esser ${ }^{11}$. Carlos Fritzsche ${ }^{12}$. Annette Haberl ${ }^{9} \cdot$ Dirk Schürmann $^{16}$. \\ Olaf Degen ${ }^{18}$. Heinz-August Horst ${ }^{19}$. Christian Hoffmann ${ }^{13} \cdot$ Björn Jensen ${ }^{14}$. Carolynne Schwarze-Zander ${ }^{7}$. \\ Martin Platten $^{15}$. Gerd Fätkenheuer ${ }^{1,2}$. Daniel Schmidt ${ }^{3,16}$. Barbara Gunsenheimer-Bartmeyer ${ }^{3}$. \\ Jörg Janne Vehreschild ${ }^{1,2,17}$. On behalf of the ClinSurv Study Group
}

Received: 25 January 2020 / Accepted: 21 June 2020 / Published online: 1 July 2020

(c) The Author(s) 2020, corrected publication 2021

\begin{abstract}
Objective Combination antiretroviral therapy (cART) has markedly increased survival and quality of life in people living with HIV. With the advent of new treatment options, including single-tablet regimens, durability and efficacy of first-line cART regimens are evolving.

Methods We analyzed data from the prospective multicenter German Clinical Surveillance of HIV Disease (ClinSurv) cohort of the Robert-Koch Institute. Kaplan-Meier and Cox proportional hazards models were run to examine the factors associated with treatment modification. Recovery after treatment initiation was analyzed comparing pre-cART viral load and CD4+ T-cell counts with follow-up data.

Results We included 8788 patients who initiated cART between 2005 and 2017. The sample population was predominantly male $(n=7040 ; 80.1 \%)$, of whom $4470(63.5 \%)$ were reporting sex with men as the transmission risk factor. Overall, 4210 (47.9\%) patients modified their first-line cART after a median time of 63 months (IQR 59-66). Regimens containing integrase strand transfer inhibitors (INSTI) were associated with significantly lower rates of treatment modification (adjusted hazard ratio 0.44 ; $95 \%$ CI 0.39-0.50) compared to protease inhibitor (PI)-based regimens. We found a decreased durability of first-line cART significantly associated with being female, a low CD4+ T-cell count, cART initiation in the later period (2011-2017), being on a multi-tablet regimen (MTR).

Conclusions Drug class and MTRs are significantly associated with treatment modification. INSTI-based regimens showed to be superior compared to PI-based regimens in terms of durability.
\end{abstract}

Keywords HIV · cART $\cdot$ Treatment modification · First-line regimen

Melanie Stecher and Philipp Schommers contributed equally to this work.

Electronic supplementary material The online version of this article (https://doi.org/10.1007/s15010-020-01469-6) contains supplementary material, which is available to authorized users.

Melanie Stecher

melanie.stecher@uk-koeln.de

Jörg Janne Vehreschild joerg.vehreschild@uk-koeln.de

Extended author information available on the last page of the article

\section{Introduction}

Combination antiretroviral therapy (cART) has improved markedly over the past decades. Today, people living with HIV (PLWH) can mostly be treated with safe and well-tolerated cART leading to a long-term suppression of viremia [1], which results in a significant reduction of morbidity and mortality in PLWH. New drug combinations are available as once-daily or single-tablet regimens (STR) that improve adherence to cART and consequently lead to successful suppression of viremia [2]. An effective virological control and immunological reconstitution is crucial for therapeutic long-term management in PLWH. Reports from the United 
States of America (USA) of the early cART era found a median duration of the first-line cART regimen between 1 and 3 years, depending on the observed periods ranging from 1996 to 2009 [3-6]. A recent study found that this time was extended to almost 5 years in the period between 2008 and 2011 [7]. Data from Australia showed a trend to fewer treatment modifications in recent years and demonstrated a stable rate of first-line treatment modifications, comparable to other cohorts [8]. Various studies found factors that are believed to lead to an earlier modification of the initial cART, including treatment with a protease inhibitor (PI), a high baseline HIV RNA level, and multiple-tablet regimens (MTR) as well as not receiving a once-daily cART or STR $[3,9,10]$.

While several of these factors are conclusive and have been proven by different cohort studies, most of these studies have been done before the introduction of integrase strand transfer inhibitors (INSTI). The first INSTI, raltegravir (RAL), was approved by the Food and Drug Administration (FDA) in 2007 [11]. It was followed by elvitegravir (EVG), dolutegravir (DTG) [12], and bictegravir (BIC) [13], which were approved by the FDA in 2012, 2013, and 2018, respectively, and were subsequently approved in Germany [14]. In 2018, INSTI-based regimens for first-line treatment were recommended, among others, by the International Antiviral Society (IAS)-USA, the European AIDS Clinical Society (EACS), and German Austrian AIDS Society $[1,15,16]$. However, there are limited data on reasons to modify firstline regimens in the era of novel cART regimens in routine clinical care conditions.

Therefore, we examined the durability of different firstline cART regimens and characteristics of those who modified first-line cART in a real-world setting. We also aimed to describe the characteristics of those who achieved viral suppression after cART initiation, as found in the national German Clinical Surveillance of HIV Disease Cohort (ClinSurv) in Germany between 2005 and 2017.

\section{Methods}

\section{Study design}

This study was planned and conducted by the academic and public sector researchers of the University Hospital of Cologne and German Center for Infection Research. We conducted a retrospective analysis of PLWH enrolled in the German national ClinSurv HIV cohort of the Robert Koch Institute (RKI), an ongoing, long-term observational multicenter cohort initiated in 1999. Details of the ClinSurv HIV cohort have been published previously [17]. In brief, 15 German University Hospitals and specialized clinical HIV treatment centers contribute comprehensive data on treatment and outcomes to the ClinSurv cohort. Data for ClinSurv are collected upon enrollment and are updated biannually based on all visits to the participating centers.

\section{Study population}

PLWH enrolled in ClinSurv from January 2005 through June 2017 were eligible for our analysis if they initiated their first-line therapy during this time period, were older than 18 years, and had both, an available pre-cART CD4+ T-cell count and viral load, yielding 8788 participants. Patients who received their first-line treatment in the setting of a clinical study were excluded from this analysis.

\section{Definition of the endpoint}

The first endpoint was defined as the date when the firstline drug class was changed to another class during followup. Modification was not counted if individuals alternated the dosage or used other drugs within the same drug class or if they interrupted the first-line drug class. The second outcome was defined as immunological recovery after 12 months of first-line treatment. Patients were censored if they died during the study period, failed to follow-up, or reached the end of the observation period before experiencing the endpoint. The epidemiological trends were evaluated between 2005 and 2017 (date of starting the respective cART). Moreover, we compared an early period (2005-2010) and late period (2011-2017), with the latter representing the period in which current INSTI regimens and STRs become available.

\section{Statistical analyses}

Baseline characteristics of included patients were reported as absolute numbers with percentage and median with interquartile range (IQR), as appropriate. Patients were compared during the period 2005-2010 (early period) and 2011-2017 (late period), using the Chi-square test.

We analyzed two different outcomes. First, we examined the durability of the first-line regimen, defined as switching the first-line cART drug class. Second, we analyzed viral suppression after cART initiation, defined as achieving low or undetectable plasma HIV RNA $(<200$ copies $/ \mathrm{mL})$ after 12 months ( \pm 6 months). The Kaplan-Meier (KM) method was used to examine factors independently associated with the durability of first-line cART. Differences between subgroups were compared by the log-rank test. The presence of multicollinearity problems was assessed among the explanatory variables using the Tolerance and Variance Inflating Factor [18]. A multivariable Cox regression model was used to identify the factors associated with the durability of the first-line cART regimen. We used backward selection 
eliminating variables with $p>0.2$ to reach the simplest model that explained the data. Our final multivariable model was adjusted for sex, transmission risk group, pre-cART CD4+ T-cell count and viral load, first-line drug class, antiretroviral drugs that were included in the first-line regimen (TDF/FTC in combination with EFV, DRV/r, LPV/r, ATV/r, RAL, NVP, RPV, DTF or Others), the tablet regimen (single versus multi tablet regimen), and the period of cART initiation (2005-2010 and 2011-2017). Hazard ratio (HR) and adjusted hazard ratio (aHR) with 95\% confidence intervals $(\mathrm{CI})$ were reported to measure the strength and association between variables.

We further analyzed immunological recovery after firstline initiation. Pre-cART viral load and CD4+ T-cell count were compared to the closest measurement of month 12 ( \pm 6 months). $P$ values of $<0.05$ were considered statistically significant. All analyses were compiled using STATA (Stata Statistical Software: Release 14. College Station, StataCorp LP, TX, USA).

\section{Ethical consideration}

The RKI is the national public health institute and is responsible for disease prevention and control in Germany. The Federal Commissioner for Data Protection is the responsible entity for studies conducted by the RKI. All HIV infections are reported to the RKI as a statutory duty for anonymous notification, implemented by the national Protection against Infection Act. The data collected in the ClinSurv cohort are generated during routine care. In this scenario, no informed consent or permission for secondary analysis of the anonymized data was required. This study was performed in accordance with the Declaration of Helsinki.

\section{Results}

\section{Study population}

Between January 2005 and June 2017, a total of 8788 PLWH met the inclusion criteria and were eligible for our analyses (Figure S1). Baseline characteristics are presented in Table 1 . The median age was 38 years (IQR 31-46), and patients were predominantly male $(n=7040 ; 80.1 \%)$. Among male patients, 4470 (63.5\%) reported sex with men (MSM) as the main transmission risk factor. In the total study population, the median pretreatment CD4+ T-cell count was 241 cells $/ \mu \mathrm{L}$ (IQR $111-369$ cells $/ \mu \mathrm{L}$ ), and $39.6 \%$ of patients had a CD4+ T-cell count below 200 cells $/ \mu \mathrm{L}$. The median pretreatment HIV RNA was 65,000 copies $/ \mathrm{mL}$ (IQR $13,903-213,000$ ), and $38.9 \%$ of patients had a pretreatment viral load greater than 100,000 copies $/ \mathrm{mL}$. A total of 338 (3.8\%) patients died over the observation period, of whom
177 (52.4\%) were on a PI-based regimen, 105 (31.1\%) on a nucleotide reverse-transcriptase inhibitor (NRTI)/non-nucleotide reverse-transcriptase inhibitor (NNRTI) regimen, and $22(10.1 \%)$ on an INSTI-based regimen.

The most common prescribed first-line cART regimens were TDF/FTC/EFV $(n=1734 / 8788 ; 19.7 \%)$ and TDF/FTC/ $\mathrm{DRV} / \mathrm{r}(n=1180 / 8788 ; 13.4 \%)$. Within the early period of 2005-2010, TDF/FTC/EFV accounted for almost one third $(n=1285 / 4450 ; 28.2 \%)$ of all prescribed cARTs, while $\mathrm{TDF} / \mathrm{FTC} / \mathrm{DRV} / \mathrm{r}(n=860 / 4238 ; 20.3 \%)$ was the most frequent cART during the late period of 2011-2017. Most of the common first-line drug class combinations were NRTI/PI/boosted ( $n=3682 / 8788 ; 41.9 \%)$, NRTI/NNRTI $(n=2951 / 8788 ; 33.6 \%)$, and NRTI/INSTI $(n=1676 / 8788$; $19.1 \%$ ), shown in Table 1 . Since 2010, treatment initiation with NRTI/PI/boosted or NRTI/NNRTI decreased continuously and dropped below 5\% in 2017. In comparison, treatment initiation with INSTI-based regimens increased constantly since 2008 and amounted to $85 \%$ of all patients initiating cART in 2017. Changes over time are shown in detail in Fig. 1.

\section{Durability of the first-line CART regimen}

During 44,439 patient-years of follow-up and a median follow-up time of 3.83 years (IQR 1.30-7.81) per patient, the overall rate of first-line cART modification was 25 per 100 person-years. In total, 4210 (47.9\%) patients modified their first-line therapy during follow-up. The median durability was 63 months (IQR 59-66), and was significantly longer in the early period compared to the late period (68 months, 95\% CI $64-72$ vs. 52 months, 95\% CI 48-55; log-rank test $p=0.002)($ Table S1).

The reason for modifying first-line therapy was recorded in $3597 / 4210(85.4 \%)$ patients. The most commonly reported causes in men and women were side effects of drugs 792/3597 (22.0\%), simplification of therapy 394/3597 (11.0\%), patients' choice $267 / 3597$ (7.4\%), decision of the responsible physician 259/3597 (7.2\%), non-adherence 212/3597 (5.9\%), comorbidities 136/3597 (3.8\%), and virological failure 133/3597 (3.7\%). Among women, side effects of drugs 152/588 (25.9\%), patients' choice 75/588 (12.8\%), simplification of therapy 70/588 (11.9\%), and pregnancy $51 / 588(8.7 \%)$ were the most common causes to modify first-line therapy.

Physicians' choice to modify first-line therapy was reported in $4.9 \%(128 / 1609)$ of patients during the early period and increased to $8.1 \%$ (131/2601) during the late period. The percentage of patients who modified first-line cART due to the simplification of therapy was lower in the early period than in the late period $(192 / 2601 ; 7.4 \%$ vs. 202/1609; 12.6\%). 
Table 1 Overall patient characteristics and comparing characteristics during the early (2005-2010) and late period (2011-2017)

\begin{tabular}{|c|c|c|c|c|}
\hline & Patient characteristics, $n(\%)$ & 2005-2010, $n(\%)$ & 2011-2017, n(\%) & $p$ value* \\
\hline Total & $8788(100)$ & $4550(51.8)$ & $4238(48.2)$ & \\
\hline Age (median, IQR) & $38(31-46)$ & $38(31-46)$ & $38(30-47)$ & 0.025 \\
\hline $18-39$ & $4629(52.7)$ & $2436(55.5)$ & $2193(53.6)$ & \\
\hline $40-69$ & $3770(42.9)$ & $1921(43.8)$ & $1849(45.2)$ & \\
\hline$\geq 70$ & $77(0.9)$ & $30(0.7)$ & $47(1.1)$ & \\
\hline Sex & & & & 0.109 \\
\hline Female & $1748(19.9)$ & $935(20.5)$ & $813(19.2)$ & \\
\hline Male & $7040(80.1)$ & $3615(79.5$ & $3425(80.8)$ & \\
\hline Region of origin & & & & $<0.001$ \\
\hline Germany & $6046(68.8)$ & $3218(70.7)$ & $2828(66.7)$ & \\
\hline Europe & $993(11.3)$ & $441(9.7)$ & $552(13.0)$ & \\
\hline Middle East & $90(1.0)$ & $39(0.9)$ & $51(1.2)$ & \\
\hline Sub-Saharan Africa & $1005(11.4)$ & $533(11.7)$ & $472(11.1)$ & \\
\hline Asia, Australia and New-Zealand & $254(2.9)$ & $152(3.3)$ & $102(2.4)$ & \\
\hline North and Latin America & $222(2.5)$ & $118(2.6)$ & $104(2.5)$ & \\
\hline Others/unknown & $178(2.0)$ & $49(1.1)$ & $129(3.0)$ & \\
\hline Risk group & & & & $<0.001$ \\
\hline MSM & $4470(50.9)$ & $2273(50.0)$ & $2197(51.8)$ & \\
\hline HTS & $1467(16.7)$ & $279(6.1)$ & $172(4.1)$ & \\
\hline ENDEMIC & $1171(13.3)$ & $748(16.4)$ & $719(17.0)$ & \\
\hline PWID & $451(5.1)$ & $646(16.4)$ & $525(12.4)$ & \\
\hline Other/unknown & $1229(14.0)$ & $604(13.3)$ & $625(14.7)$ & \\
\hline Pre-cART CD4+ T-cell count $(\mu \mathrm{L})$ & & & & $<0.001$ \\
\hline (Median, IQR) & $241(111-369)$ & $215(102-320)$ & $280(124-425)$ & \\
\hline$<200$ & 3479 (39.6) & $2014(45.9)$ & 1465 (35.6) & \\
\hline $200-349$ & $2631(29.9)$ & $1503(34.3)$ & $1128(27.4)$ & \\
\hline $350-499$ & $1399(15.9)$ & $575(13.1)$ & $824(20.0)$ & \\
\hline$\geq 500$ & $992(11.3)$ & $295(6.7)$ & $697(16.9)$ & \\
\hline $\begin{array}{l}\text { Pre-cART HIV-1 RNA viral load (copies/ } \\
\text { mL) (median, IQR) }\end{array}$ & $65,000(13,903-213,000)$ & $68,575(15,276-223,904)$ & $60,400(12,500-205,317)$ & 0.114 \\
\hline$<200$ & $196(2.2)$ & $104(2.5)$ & $924(2.3)$ & \\
\hline $201-5000$ & $939(10.7)$ & $494(11.7)$ & $445(11.3)$ & \\
\hline $5001-100,000$ & $3638(41.4)$ & $1838(43.6)$ & $1800(45.7)$ & \\
\hline 100,001-1 Mio & $2911(33.1)$ & $1550(36.8)$ & $1361(34.5)$ & \\
\hline$>1$ Mio & $474(5.4)$ & $229(5.4)$ & $245(6.2)$ & \\
\hline Durability of first-line in months (IQR) & $63(59-66)$ & $68(64-72)$ & $52(48-55)$ & $<0.001$ \\
\hline First-line drug class & & & & $<0.001$ \\
\hline NRTI/PI/boosted & $3682(41.9)$ & $2140(47.0)$ & $1542(36.4)$ & \\
\hline NRTI/NNRTI & $2951(33.6)$ & $1945(42.7)$ & $1006(23.7)$ & \\
\hline NRTI/INSTI & $1676(19.1)$ & $146(3.2)$ & $1530(36.1)$ & \\
\hline Others & $479(5.5)(4.4)$ & $319(7.0)$ & $160(3.8)$ & \\
\hline Substance of the first-line regimen & & & & $<0.001$ \\
\hline TDF/FTC/EFV & $1734(19.7)$ & $1285(28.2)$ & $449(10.6)$ & \\
\hline $\mathrm{TDF} / \mathrm{FTC} / \mathrm{DRV} / \mathrm{r}$ & $1180(13.4)$ & $320(7.0)$ & $860(20.3)$ & \\
\hline TDF/FTC/LPV/r & $863(9.8)$ & $708(15.6)$ & $155(3.7)$ & \\
\hline $\mathrm{TDF} / \mathrm{FTC} / \mathrm{ATV} / \mathrm{r}$ & $655(7.5)$ & $301(6.6)$ & $354(8.4)$ & \\
\hline TDF/FTC/RAL & $515(5.9)$ & $116(2.5)$ & $399(9.4)$ & \\
\hline TDF/FTC/NVP & $469(5.3)$ & $348(7.6)$ & $121(2.9)$ & \\
\hline TDF/FTC/RPV & $367(4.2)$ & $18(0.4)$ & $349(8.2)$ & \\
\hline TDF/FTC/DTG & $352(4.0)$ & $15(0.3)$ & $337(8.0)$ & \\
\hline
\end{tabular}


Table 1 (continued)

\begin{tabular}{|c|c|c|c|c|}
\hline & Patient characteristics, $n(\%)$ & 2005-2010, $n(\%)$ & 2011-2017, $n(\%)$ & $p$ value* \\
\hline Others & $2653(30.2)$ & 1439 (31.6) & $1214(28.6)$ & \\
\hline Number of tablets per day & & & & $<0.001$ \\
\hline 1 & $1113(13)$ & $93(2.1)$ & $1020(24.5)$ & \\
\hline $2-3$ & $4614(53.7)$ & $2283(51.6)$ & $2331(55.9)$ & \\
\hline $4-9$ & $2796(32.5)$ & $1983(44.8)$ & $813(19.5)$ & \\
\hline$\geq 10$ & $69(0.8)$ & $66(1.5)$ & $3(0.1)$ & \\
\hline Single tablet regimen & & & & 0.272 \\
\hline STR & $2472(28.1)$ & 1303 (28.6) & 1169 (27.6) & \\
\hline MTR & $6316(71.9)$ & $3247(71.4)$ & $3069(72.4)$ & \\
\hline Regimen with INSTIs & & & & $<0.001$ \\
\hline RAL & $653(7.4)$ & $144(88.9)$ & 509 (31.9) & \\
\hline DTG & $746(8.5)$ & $17(10.5)$ & 729 (45.7) & \\
\hline EVG & $359(4.1)$ & $1(0.6)$ & $358(22.4)$ & \\
\hline Tablet intake & & & & $<0.001$ \\
\hline Once per day & $6063(69.0)$ & $2547(41.3)$ & $3516(83.0)$ & \\
\hline Twice per day & $2529(28.8)$ & $1878(41.3)$ & $651(15.4)$ & \\
\hline Reason for discontinuing first-line therapy & & & & $<0.001$ \\
\hline Side effects of drugs & $792(9.0)$ & $514(11.3)$ & $278(6.6)$ & \\
\hline Simplification of therapy & $394(4.5)$ & $192(4.2)$ & $202(4.8)$ & \\
\hline Patients' choice & $267(3.0)$ & $168(3.7)$ & $99(2.3)$ & \\
\hline Decision of the responsible physician & $259(2.9)$ & $128(2.8)$ & $131(3.1)$ & \\
\hline Non-adherence & $212(2.4)$ & $120(2.6)$ & $92(2.2)$ & \\
\hline Concomitant diseases & $136(1.5)$ & $80(1.8)$ & $56(1.3)$ & \\
\hline Virological failure & $133(1.5)$ & $85(1.9)$ & $48(1.1)$ & \\
\hline Others & $462(5.3)$ & $276(6.1)$ & $186(4.4)$ & \\
\hline
\end{tabular}

$p$ values of $<0.05$ in bold depict significant results

Risk group: MSM men who have sex with men, HTS heterosexual, ENDEMIC recent immigration from a country with a high HIV prevalence $>1 \%, P W I D$ people who inject drugs. First-line drug class: NRTI nucleoside reverse- transcriptase inhibitor, NNRTI non-nucleoside reverse-transcriptase inhibitors, INSTI integrase strand transfer inhibitors, $P I$ protease inhibitor. Substance of first-line regimen: $T D F$ tenofovir, $F T C$ emtricitabine, $E F V$ efavirenz, $D R V$ darunavir, $A T V$ atazanavir, $R A L$ raltegravir, $N V P$ nevirapine, $R P V$ rilpivirine, $D T G$ dolutegravir. Regime: STR single-tablet regimen, $M T R$ multi-tablet regimen. First-line with INSTI regimen: $R A L$ raltegravir, $E V G$ elvitegravir, $D L G$ dolutegravir

*Chi-Square test $(p<0.05)$

Of the 4210 patients who modified their first-line cART, $21.5 \%$ (701/3259) switched from a MTR to a STR. The proportion increased from $19.4 \%$ (384/1981) in the early period to $24.8 \%(317 / 1278)$ during the late period $(p<0.001)$. A total of $31.3 \%(1084 / 3464)$ switched from a non-INSTI towards an INSTI-based regimen, the proportion increased from $24.6 \%(564 / 2297)$ to $44.6 \%(520 / 1167 ; p<0.001)$ comparing the early to the late period.

\section{Factors associated with first-line cART durability}

Time-to-event analyses revealed prognostic factors to modify the first-line regimen, illustrated in the KM plots in Fig. $2 \mathrm{a}-\mathrm{d}$ and Supplementary Figures S2 A and B and Table S1. For the duration of the first-line regimens, significant differences were identified among sex $(p<0.001)$, different drug classes $(p<0.001)$, and the year of cART initiation $(p=0.002)$. The median durability of the first-line regimen was significantly shorter in patients on MTR than in patients on STR (median 51 months, 95\% CI 47-53 vs. 93 months, 95\% CI 87-97; $p<0.001$ ) (Table S1). KM analyses also revealed an increasing trend of first-line cART modification with a lower precART CD4+ T-cell count $(<350 \mu \mathrm{L})(p<0.001)$. These differences remained significant in the adjusted multivariable Cox regression model.

Women were more likely to modify the first-line regimen than men (aHR 1.24; 95\% CI 1.12-1.37). Patients on a STR were significantly less likely to modify the first-line regimen compared to patients on an MTR (aHR 0.91; 95\% CI 0.70-0.94). In addition, the frequency of tablet intake twice daily compared to once daily was significantly associated with treatment modification (aHR 1.34; 95\% CI 1.22-1.48). 


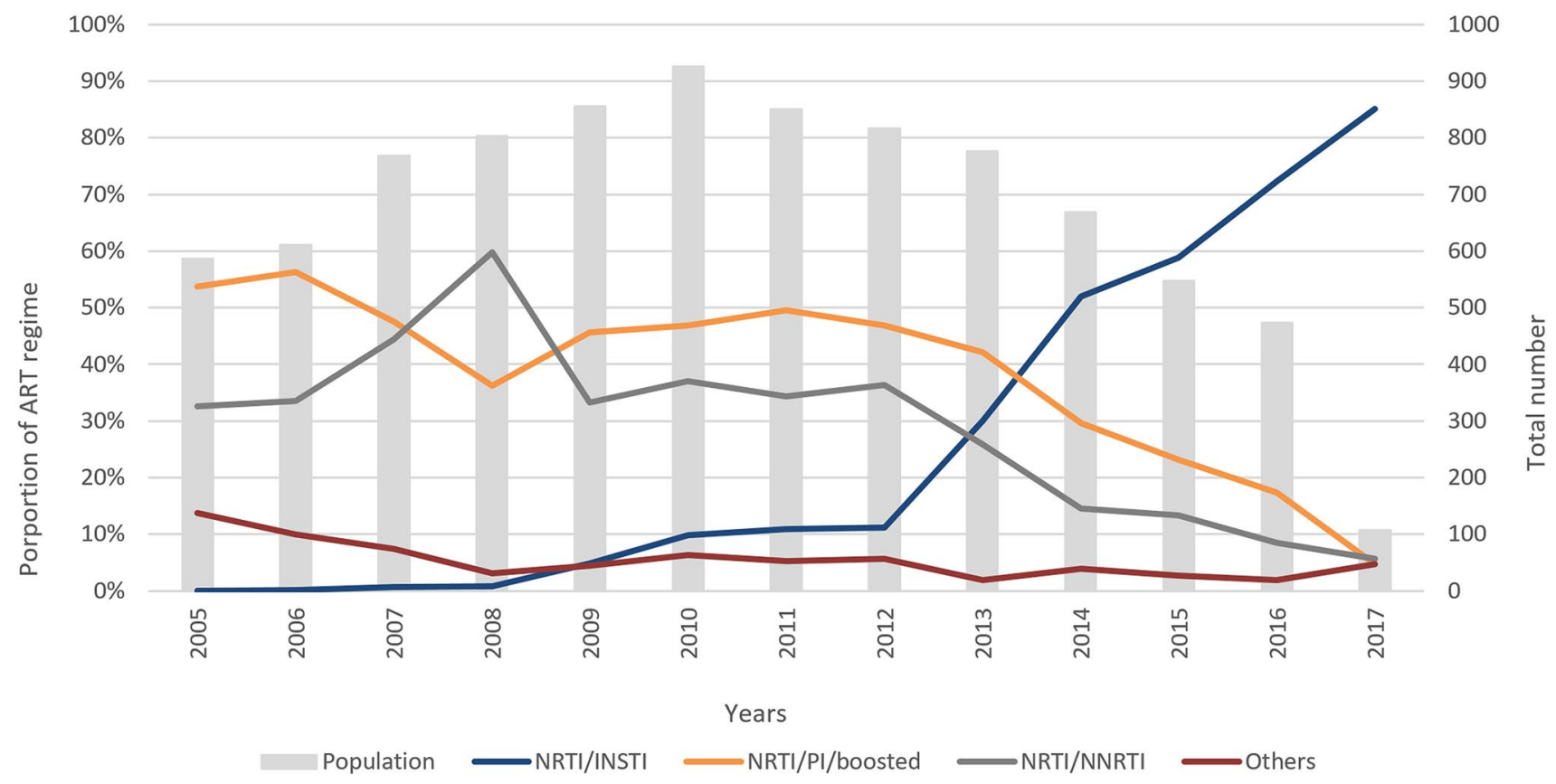

Fig. 1 First-line cART regimen and total number of patients over time. Proportions of each cART regimen (vertical axis on the left side) are shown in three lines. NRTI/INSTI (nucleoside reverse-transcriptase inhibitor/integrase strand transfer inhibitor) (blue), NRTI/ $\mathrm{PI} /$ boosted (non-nucleoside reverse-transcriptase inhibitors/protease

We found that being on a NRTI/NNRTI (aHR 0.75; 95\% CI 0.67-0.84) or a NRTI/INSTI (aHR 0.44; 95\% CI 0.39-0.50) first-line regimen was associated with lower rates of modification, compared to being on a NRTI/PI/boosted first-line regimen. Modification of first-line regimen increased in the late period (2011-2017) compared to the early period (2005-2010) (aHR 1.45; 95\% CI 1.33-1.58) (Table 2).

We also identified significant differences for modification of the discontinuation of first-line cART among transmission risk groups and different INSTI regimens. Patients on RAL and EVG were significantly more likely to modify therapy compared to those on DTG (HR 2.01; 95\% CI 1.59-2.53 and HR 1.46; 95\% CI 1.08-1.96, respectively). However, the transmission risk group did not remain significant in the adjusted multivariable Cox regression model, and INSTI regimens were excluded due to multicollinearity. Uni-and multivariable analyses were also performed separately for the early and late period. Details are displayed in supplementary table S2-S4.

\section{Antiviral efficacy and immunological recovery after first-line initiation}

Among patients with an available HIV-1 RNA assessment at month 12 ( \pm 6 months), 5745/6089 (95.4\%) achieved viral suppression $(<200$ copies $/ \mathrm{mL})$. inhibitor/boosted) (orange), NRTI/NNRTI (nucleoside reverse-transcriptase inhibitor/non-nucleoside reverse-transcriptase inhibitors) (gray), and others (red). The gray bars representing the total number of patients starting cART in the respective year (vertical axis on the right side)

Pre-cART viral load was significantly higher in patients who modified their first-line cART regimen within 12 months ( \pm 6 months) (median 70,324 copies/mL; IQR $12,812-257,914$ copies $/ \mathrm{mL}$ ) than in patients who remained on their first-line regimen (median 63,126 copies/mL; IQR $14,078-200,000 ; p=0.013$ ). In the whole group, the median decrease in viral load after 12 months on cART was $3.4 \mathrm{log}$ copies/mL (IQR 2.8-4.0 log copies/mL) and was greater in those who remained on their first-line cART than in patients who modified the initial treatment (4.0 vs. $3.5 \mathrm{log}$ copies/ $\mathrm{mL}$, respectively). The overall pre-cART CD4+ T-cell count increased with a median gain of 205 cells/ $\mu \mathrm{L}$ (IQR 180-374 cells $/ \mu \mathrm{L})$ at month 12 ( \pm 6 months), a relative increase of $84 \%$. No differences were observed between individuals who remained on their first-line regimen compared to those who modified their first-line regimen $(p=0.843)$.

\section{Discussion}

This study examined key factors for durability of first-line cART treatment in routine clinical care conditions across Germany. We found several factors that were associated with a shorter durability of first-line cART in the multivariable analysis including being female, low CD4+ T-cell counts at the beginning of treatment, PI-based cART, MTR, cART 

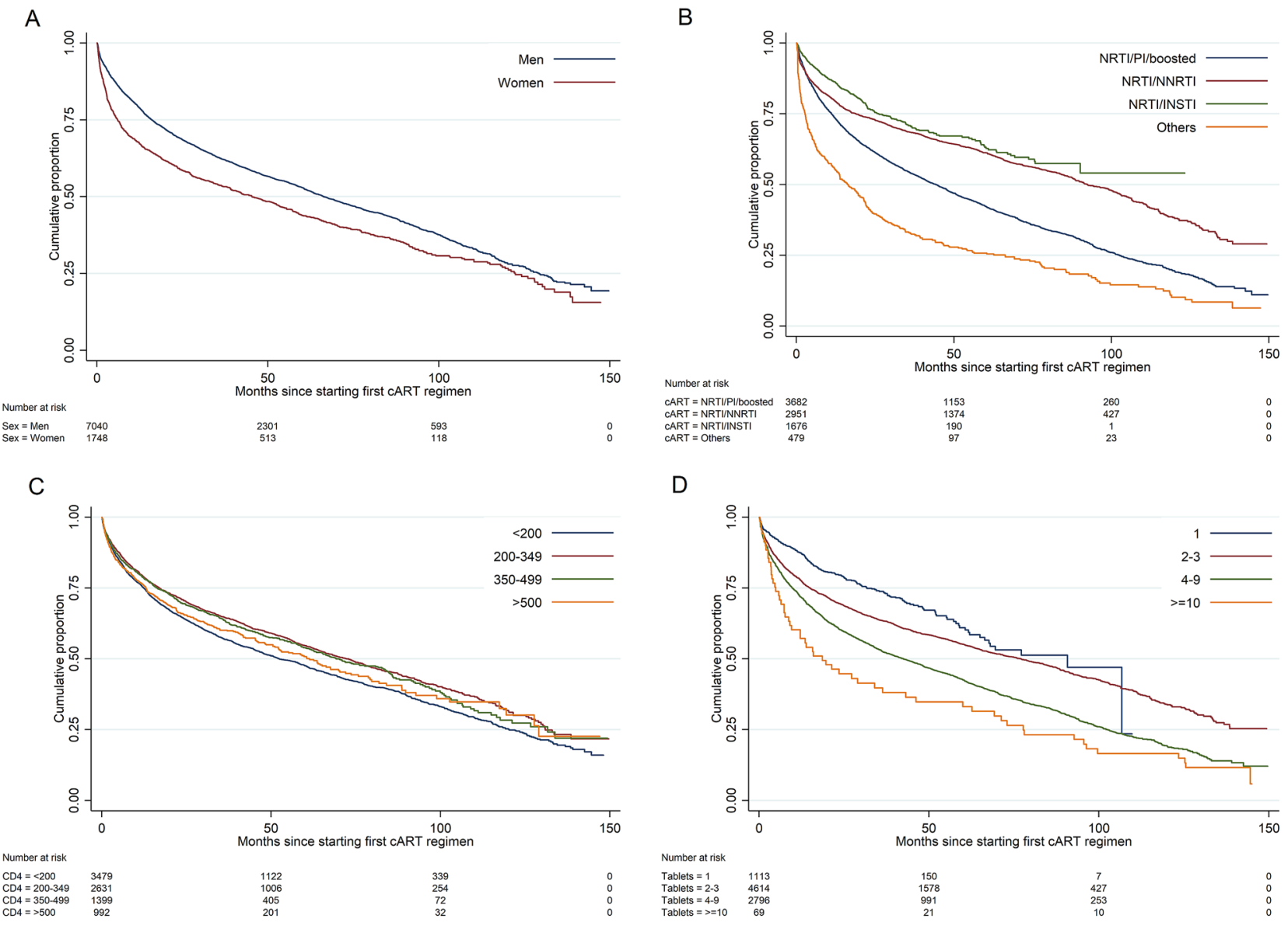

D

Fig. 2 Unadjusted cumulative proportion of first-line cART durability. a Sex, b first-line drug class: NRTI; nucleoside reverse- transcriptase inhibitor, NNRTI; non-nucleoside reverse-transcriptase

initiation in the late period (2011-2017), and tablet intake more than once a day.

The significant difference in sex, as well as in people presenting with low pre-cART CD4+ T-cell count, may be related to non-adherence that is more frequently seen in female PLWH and individuals originating from sub-Saharan Africa, as well as in late presenters [19]. In our study population, most people from sub-Saharan Africa were women (70\%). In addition, pregnancies, which accounted for about $10 \%$ of reported reasons for treatment modification, might also contribute to the lower durability of cART that is seen in women [20].

Interestingly, in our study, there was no tendency towards a better durability of first-line therapy in recent years as it was found by other studies [7, 8]. The most common causes of modification in our cohort were side effects of drugs, simplification of therapy, and patient's choice. Thus, we hypothesize that the newer INSTI-class and additional STR

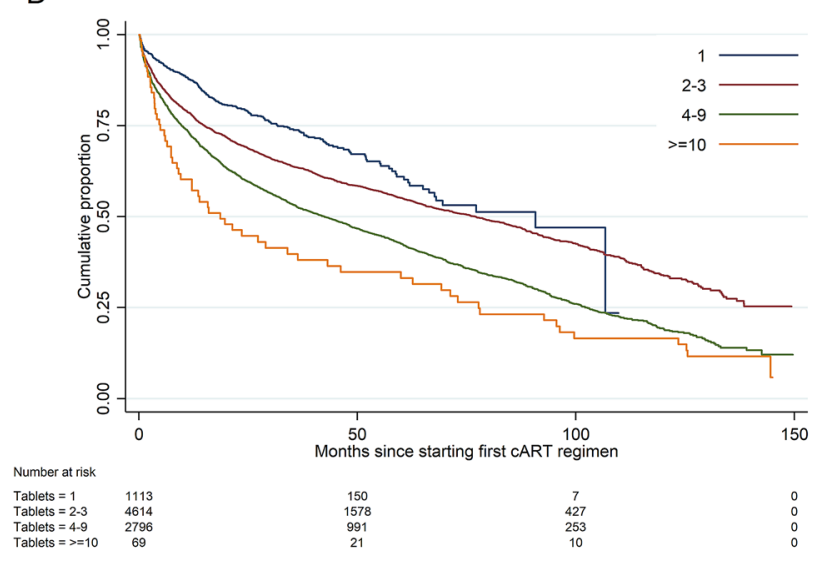

inhibitors INSTI; integrase inhibitor, PI; protease inhibitor. c PrecART CD4+ T-cell count and $\mathbf{d}$ number of tablets of the first-line cART regimen per day

options in the late period offered an interesting alternative for patients and physicians, contributing to higher rates of modification [21]. This is confirmed by our observation that physicians' choice as well as simplification of therapy as reasons for treatment switch were significantly more frequent in the late period between 2011 and 2017.

Most subjects, especially in the early period, received PI-based cART regimens, which showed a higher risk for first-line modification compared to NNRTI- or INSTI-based regimens. These results are in line with previous studies, showing an inferiority of PI-based regimens compared to NNRTI- or INSTI-based regimens in terms of treatment $[22,23]$. This might be due to more interactions with other medications and higher rates of side effects. However, higher costs and strategic changes in therapy could also partially explain higher rates of modification of PI-based regimens. In addition, PI-based STR has only been available since 2017, so they may be underrepresented in our cohort. 
Table 2 Associations between baseline characteristics and first-line cART modification between 2005 and 2017

\begin{tabular}{|c|c|c|c|c|}
\hline & \multicolumn{2}{|c|}{ Univariable model* } & \multicolumn{2}{|c|}{ Mutivariable model* } \\
\hline & HR (95\% CI) & $p$ value & aHR (95\% CI) & $p$ value \\
\hline \multicolumn{5}{|l|}{ Age } \\
\hline \multicolumn{5}{|l|}{$18-39$} \\
\hline $40-69$ & $1.02(0.96-1.09)$ & 0.519 & & \\
\hline$\geq 70$ & $1.06(0.77-1.48)$ & 0.711 & & \\
\hline \multicolumn{5}{|l|}{ Sex } \\
\hline Female & $1.32(0.23-1.42)$ & $<0.001$ & $1.24(1.12-1.37)$ & $<0.001$ \\
\hline \multicolumn{5}{|l|}{ Male } \\
\hline \multicolumn{5}{|l|}{ Region of origin } \\
\hline \multicolumn{5}{|l|}{ Germany } \\
\hline Europe & $1.12(1.02-1.24)$ & $\mathbf{0 . 0 2 0}$ & $1.06(0.96-1.19)$ & 0.256 \\
\hline Middle East & $1.14(0.85-1.52)$ & 0.392 & $1.17(0.86-1.95)$ & 0.314 \\
\hline Sub-Saharan Africa & $1.19(1.09-1.31)$ & $<0.001$ & $0.94(0.73-1.20)$ & 0.621 \\
\hline Asia, Australia and New-Zealand & $1.05(0.88-1.25)$ & 0.613 & $0.98(0.79-1.23)$ & 0.915 \\
\hline North and Latin America & $0.89(0.72-1.09)$ & 0.521 & $0.90(0.72-1.13)$ & 0.371 \\
\hline Others/unknown & $1.40(1.11-1.69)$ & 0.003 & $1.39(1.09-1.77)$ & 0.007 \\
\hline \multicolumn{5}{|l|}{ Transmission risk group } \\
\hline \multicolumn{5}{|l|}{ MSM } \\
\hline PWID & $1.33(1.16-1.52)$ & $<0.001$ & $1.04(0.90-1.20)$ & 0.597 \\
\hline HTS & $1.09(1.00-1.19)$ & $\mathbf{0 . 0 3 9}$ & $0.98(0.88-1.09)$ & 0.726 \\
\hline ENDEMIC & $1.26(1.15-1.38)$ & $<0.001$ & $1.03(0.81-1.31)$ & 0.823 \\
\hline Other/unknown & $1.18(1.08-1.30)$ & $<0.001$ & $1.02(0.92-1.13)$ & 0.751 \\
\hline \multicolumn{5}{|l|}{ Pre-cART CD4+ T-cell count $(\mu \mathrm{L})$} \\
\hline \multicolumn{5}{|l|}{$<200$} \\
\hline $200-349$ & $0.82(0.76-0.88)$ & $<0.001$ & $0.92(0.85-0.99)$ & $\mathbf{0 . 0 3 7}$ \\
\hline $350-499$ & $0.85(0.77-0.93)$ & $<0.001$ & $0.97(0.88-1.07)$ & 0.557 \\
\hline$\geq 500$ & $0.94(0.84-1.05)$ & 0.242 & $1.07(0.95-1.21)$ & 0.250 \\
\hline \multicolumn{5}{|c|}{ Pre-cART HIV-1 RNA viral load (copies/mL) } \\
\hline$<200$ & $0.97(0.79-1.20)$ & 0.789 & $0.90(0.71-1.14)$ & 0.377 \\
\hline $201-5000$ & $0.95(0.86-1.06)$ & 0.373 & $0.82(0.66-1.02)$ & 0.079 \\
\hline $5001-100,000$ & $0.85(0.79-0.91)$ & $<0.001$ & $0.93(0.75-1.16)$ & 0.509 \\
\hline \multicolumn{5}{|l|}{$>100,000$} \\
\hline \multicolumn{5}{|l|}{ First-line drug class } \\
\hline \multicolumn{5}{|l|}{ NRTI/PI/boosted } \\
\hline NRTI/NNRTI & $0.61(0.57-0.65)$ & $<0.001$ & $0.75(0.67-0.84)$ & $<0.001$ \\
\hline NRTI/INSTI & $0.52(0.46-0.57)$ & $<0.001$ & $0.44(0.39-0.50)$ & $<0.001$ \\
\hline Others & $1.67(1.50-1.87)$ & $<0.001$ & $1.41(1.23-1.62)$ & $<0.001$ \\
\hline \multicolumn{5}{|l|}{ Tablet regimen } \\
\hline STR & $0.64(0.59-0.68)$ & $<0.001$ & $0.91(0.70-0.94)$ & 0.041 \\
\hline \multicolumn{5}{|l|}{ MTR } \\
\hline \multicolumn{5}{|l|}{ Year of cART initiation } \\
\hline \multicolumn{5}{|l|}{ 2005-2010 } \\
\hline 2011-2017 & $1.11(1.04-1.19)$ & 0.002 & $1.45(1.33-1.58)$ & $<0.001$ \\
\hline \multicolumn{5}{|l|}{ INSTI regimen** } \\
\hline \multicolumn{5}{|l|}{ DTG } \\
\hline RAL & $2.01(1.59-2.53)$ & $<0.001$ & & \\
\hline EVG & $1.46(1.08-1.96)$ & 0.013 & & \\
\hline
\end{tabular}


Table 2 (continued)

\begin{tabular}{llllll}
\hline & \multicolumn{2}{l}{ Univariable model* } & & \multicolumn{2}{l}{ Mutivariable model* } \\
\cline { 2 - 2 } & HR $(95 \% \mathrm{CI})$ & $p$ value & & aHR (95\% CI) & $p$ value \\
\hline $\begin{array}{l}\text { Tablet intake } \\
\text { Once per day } \\
\text { Twice per day }\end{array}$ & $1.42(1.34-1.52)$ & $<\mathbf{0 . 0 0 1}$ & & $1.34(1.22-1.48)$ & $<\mathbf{0 . 0 0 1}$ \\
\hline
\end{tabular}

$p$ values $<0.05$ in bold depict significant results

Risk group: MSM men who have sex with men, HTS heterosexual, ENDEMIC recent immigration from a country with a high HIV prevalence $>1 \%, P W I D$ people who inject drugs. First-line drug class: NRTI nucleoside reverse-transcriptase inhibitor, NNRTI non-nucleoside reverse-transcriptase inhibitors, INSTI integrase strand transfer inhibitors, $P I$ protease inhibitor. Substance of first-line regimen: $T D F$ tenofovir, FTC emtricitabine, $E F V$ efavirenz, $D R V$ darunavir, $A T V$ atazanavir, $R A L$ raltegravir, $N V P$ nevirapine, $R P V$ rilpivirine, $D T G$ dolutegravir. Tablet regimen: $S T R$ single-tablet regimen, MTR multi-tablet regimen. Firstline with INSTI regimen: $R A L$ raltegravir, $E V G$ elvitegravir, $D L G$ dolutegravir

* Results from a Cox proportional hazards model displayed with adjusted Hazard ratios (aHRs) and 95\% confidence intervals (CI)

**Variable excluded from multivariable analysis due to multicollinearity

Once-daily regimens, especially those with an STR, showed a better durability of the first-line treatment, as also shown in other studies [4, 10, 24, 25]. This effect is most likely due to the potentially improved adherence that is seen with these treatment regimens [26] and also due to the lack of further optimization options.

From 2014 onwards, INSTI-based regimens already accounted for the majority of initial treatments resulting in a maximum of $86 \%$ in the year 2017 . We found a higher risk for modification on RAL compared to EVG and DTG, mainly due to therapy simplification, which is comparable to previous studies [27, 28]. Furthermore, we found that patients who received INSTI-based regimens as their firstline treatment had the highest probability of an undetectable viremia after 12 months. Thus, these data underline the current role of INSTI-based regimens as recommended first-line treatments [1]. However, even in our study, longterm data of INSTI-based regimens are still rare.

Our study had a couple of limitations. First, these are retrospective, "real-life" data predominantly clinical HIV centers, which occupy a key position in outpatient care in the German health care system. In our analysis, patients had a median age of 38 years and were predominantly men $(80.1 \%)$, of which about one third (63.5\%) reported to be MSM. These baseline characteristics did not change notably over time comparing the early period of 2005-2010 with the late period of 2011-2017. Thus, while our study was limited to PLWH in Germany, the characteristics of patients starting their first-line antiretroviral treatment compare very well with those reported in other cohorts in the USA, France, and Australia and, therefore, seem to be representative for PLWH seen in outpatient clinics in industrialized countries [7-9]. Second, we were lacking data about severe medical preconditions such as prior AIDS-defining diseases of these patients that could have favored some cART regimens.

In conclusion, overall efficacy of first-line treatment was good, with $95.4 \%$ achieving viral suppression 1 year after starting cART. Amongst the different classes, INSTI-based regimens showed superiority in terms of durability. Thus, our data confirm the rationale of the current guidelines that recommend INSTI-based regimens as first-line treatment in HIV-infected patients. However, future studies are required to assess the efficacy and durability of these treatments over a longer period. Recent studies observed high modification rates of INSTIs, in particular for DTG, due to adverse events and weight gain $[29,30]$.

We elucidated several factors that were significantly associated with the modification of first-line regimens. The modification rates were higher during the late period, particularly among women, and in patients on an MTR. Patients at higher risk for treatment modification might require more frequent follow-up visits and better monitoring during their first-line cART regimen.

Acknowledgements Open Access funding provided by Projekt DEAL. This study was conducted and supported by the German Center for Infection Research (DZIF) [NCT02149004]. Initial results of this study were presented at the IDWeek in San Francisco, USA, 2018. We thank all patients, physicians, and study nurses of all collaborating ClinSurv-HIV Cohort study centers: Berlin: Dr. F. Bergmann, M. Warncke: Charité, University Medicine Berlin. Bonn: S. Hass: University Hospital Bonn. Düsseldorf: C. Feind: University Hospital Düsseldorf. Essen: P. Schenk-Westkamp: University Hospital Essen. Frankfurt: P. Schott: HIV Center Frankfurt. Hamburg: Prof. Dr. A. 
Plettenberg, Dr. T. Lorenzen: ifi (Institut for interdisciplinary medicine) Drs. A. Adam, K. Schewe, S. Fenske, T. Buhk, D. Radzuweit, A. Mainka: ICH (Infectious Diseases Medical Center Hamburg) Dr. O. Degen, Dr. G. Schäfer, M. Franz: University Hospital Hamburg Eppendorf. Hannover: Prof. Dr. H.G. Behrens, S. Gerschmann, R. Beider: Hannover Medical School. Kiel: Prof. Dr. H. Horst, S. Trautmann: University Hospital Schleswig-Holstein. Munich: B. Sonntag: LudwigMaximilians-University Munich.

Author contributions JV, MP, MeSt and PS designed the study. MeSt and PS analyzed and interpreted the data. CK, BGB and DS provided the data, and contributed important ideas on how to interpret the data. MeSt and PS drafted the primary draft of the manuscript. MeSt, PS, and JV revised and finalized the manuscript. All authors revised and approved the final version of the manuscript.

\section{Compliance with ethical standards}

Conflict of interest $\mathrm{CB}$ has received honoraria for consulting or educational lectures from abbvie, BMS, Gilead, Janssen, MSD and ViiV. Research grants from Dt. Leberstiftung, DZIF, Hector Stiftung and NEAT ID. JV has personal fees from Merck/MSD, Gilead, Pfizer, Astellas Pharma, Basilea, German Centre for Infection Research (DZIF), University Hospital Freiburg/Congress and Communication, Academy for Infectious Medicine, University Manchester, German Society for Infectious Diseases (DGI), Ärztekammer Nordrhein, University Hospital Aachen, Back Bay Strategies, German Society for Internal Medicine (DGIM) and grants from Merck/MSD, Gilead, Pfizer, Astellas Pharma, Basilea, German Centre for Infection Research (DZIF), German Federal Ministry of Education and Research (BMBF), (PJ-T: DLR), University of Bristol, Rigshospitalet Copenhagen. All other authors declare no conflicts of interest.

Open Access This article is licensed under a Creative Commons Attribution 4.0 International License, which permits use, sharing, adaptation, distribution and reproduction in any medium or format, as long as you give appropriate credit to the original author(s) and the source, provide a link to the Creative Commons licence, and indicate if changes were made. The images or other third party material in this article are included in the article's Creative Commons licence, unless indicated otherwise in a credit line to the material. If material is not included in the article's Creative Commons licence and your intended use is not permitted by statutory regulation or exceeds the permitted use, you will need to obtain permission directly from the copyright holder. To view a copy of this licence, visit http://creativecommons.org/licenses/by/4.0/.

\section{References}

1. Saag MS, Benson CA, Gandhi RT, Hoy JF, Landovitz RJ, Mugavero $\mathrm{MJ}$, et al. Antiretroviral drugs for treatment and prevention of HIV infection in adults: 2018 recommendations of the international antiviral society-USA panel. JAMA. 2018;320:379-96. https://doi. org/10.1001/jama.2018.8431.

2. Glass TR, Sterne JA, Schneider MP, De Geest S, Nicca D, Furrer $\mathrm{H}$, et al. Self-reported nonadherence to antiretroviral therapy as a predictor of viral failure and mortality. AIDS. 2015;29:2195-200. https://doi.org/10.1097/qad.0000000000000782.

3. Palella FJ Jr, Chmiel JS, Moorman AC, Holmberg SD. Durability and predictors of success of highly active antiretroviral therapy for ambulatory HIV-infected patients. AIDS. 2002;16:1617-26.

4. Willig JH, Abroms S, Westfall AO, Routman J, Adusumilli S, Varshney M, et al. Increased regimen durability in the era of once-daily fixed-dose combination antiretroviral therapy. AIDS. 2008;22:195160. https://doi.org/10.1097/QAD.0b013e32830efd79.
5. Juday T, Grimm K, Zoe-Powers A, Willig J, Kim E. A retrospective study of HIV antiretroviral treatment persistence in a commercially insured population in the United States. AIDS Care. 2011;23:1154 62. https://doi.org/10.1080/09540121.2010.543884.

6. Chen RY, Westfall AO, Mugavero MJ, Cloud GA, Raper JL, Chatham AG, et al. Duration of highly active antiretroviral therapy regimens. Clin Infect Dis. 2003;37:714-22. https://doi. org/10.1086/377271.

7. Sheth AN, Ofotokun I, Buchacz K, Armon C, Chmiel JS, Hart RL, et al. Antiretroviral regimen durability and success in treatmentnaive and treatment-experienced patients by year of treatment initiation, united states, 1996-2011. J Acquir Immune Defic Syndr. 1999;2016(71):47-56. https://doi.org/10.1097/qai.0000000000 000813.

8. Wright ST, Law MG, Cooper DA, Keen P, McDonald A, Middleton M, et al. Temporal trends of time to antiretroviral treatment initiation, interruption and modification: examination of patients diagnosed with advanced HIV in Australia. J Int AIDS Soc. 2015;18:19463. https://doi.org/10.7448/ias.18.1.19463.

9. Cotte L, Ferry T, Pugliese P, Valantin MA, Allavena C, Cabie A, et al. Effectiveness and tolerance of single tablet versus once daily multiple tablet regimens as first-line antiretroviral therapyresults from a large French multicenter cohort study. PLoS One. 2017;12:e0170661. https://doi.org/10.1371/journal.pone.0170661.

10. Parienti JJ, Bangsberg DR, Verdon R, Gardner EM. Better adherence with once-daily antiretroviral regimens: a meta-analysis. Clin Infect Dis. 2009;48:484-8. https://doi.org/10.1086/596482.

11. The American Society of Health-System Pharmacists. Raltegravir Potassium. 2019. https://www.drugs.com/monograph/raltegravi r-potassium.html. Accessed February 2019.

12. The American Society of Health-System Pharmacists. Dolutegravir Sodium. 2019. https://www.drugs.com/monograph/dolutegrav ir-sodium.html. Accessed Feb 2019.

13. The American Society of Health-System Pharmacists. Biktarvy Approval History. 2018. https://www.drugs.com/history/biktarvy. html. Accessed Feb 2019.

14. Bictegravir Markham A. First global approval. Drugs. 2018;78:6016. https://doi.org/10.1007/s40265-018-0896-4.

15. European AIDS Clinical Society (EACS). European guidelines for treatment of HIV-positive adults in Europe 9.1. 2018. http://www. eacsociety.org/files/2018_guidelines-9.1-english.pdf. Accessed Feb 2018.

16. Deutsche AIDS Gesellschaft. Übersicht Leitlinien zur Therapie der HIV-1-Infektion 2017. 2018. https://daignet.de/site-content/hiv-thera pie/leitlinien-1/uebersicht-leitlinien-zur-therapie-der-hiv-1-infek tion-englisch/view. Accessed Dec 2018.

17. Batzing-Feigenbaum J, Kollan C, Kuhne A, Matysiak-Klose D, Gunsenheimer-Bartmeyer B, Hamouda O. Cohort profile: the German ClinSurv HIV project-a multicentre open clinical cohort study supplementing national HIV surveillance. HIV Med. 2011;12:269-78. https://doi.org/10.1111/j.1468-1293.2010.00879.x.

18. Vatcheva KP, Lee M, McCormick JB, Rahbar MH. Multicollinearity in regression analyses conducted in epidemiologic studies. Epidemiology. 2016. https://doi.org/10.4172/2161-1165.1000227.

19. Ortego C, Huedo-Medina TB, Santos P, Rodriguez E, Sevilla L, Warren M, et al. Sex differences in adherence to highly active antiretroviral therapy: a meta-analysis. AIDS Care. 2012;24:1519 34. https://doi.org/10.1080/09540121.2012.672722.

20. Giles ML, Achhra AC, Abraham AG, Haas AD, Gill MJ, Lee MP, et al. Sex-based differences in antiretroviral therapy initiation, switching and treatment interruptions: global overview from the International Epidemiologic Databases to Evaluate AIDS (IeDEA). J Int AIDS Soc. 2018;21:e25149. https://doi.org/10.1002/jia2.25149

21. Eaton EF, Tamhane AR, Burkholder GA, Willig JH, Saag MS, Mugavero MJ. Unanticipated effects of new drug availability on 
antiretroviral durability: implications for comparative effectiveness research. Open Forum Infect Dis. 2016;3:ofw109. https://doi. org/10.1093/ofid/ofw109.

22. Eaton EF, Tamhane A, Davy-Mendez T, Mathews WC, Moore RD, Saag MS, et al. Trends in antiretroviral therapy prescription, durability and modification: new drugs, more changes, but less failure. AIDS. 2018;32:347-55. https://doi.org/10.1097/qad.0000000000 001708 .

23. Carr A, Richardson R, Liu Z. Success and failure of initial antiretroviral therapy in adults: an updated systematic review. AIDS. 2019;33:443-53. https://doi.org/10.1097/qad.0000000000002077.

24. Molina JM, Andrade-Villanueva J, Echevarria J, Chetchotisakd P, Corral J, David N, et al. Once-daily atazanavir/ritonavir versus twice-daily lopinavir/ritonavir, each in combination with tenofovir and emtricitabine, for management of antiretroviral-naive HIV1-infected patients: 48 week efficacy and safety results of the CASTLE study. Lancet. 2008;372:646-55. https://doi.org/10.1016/s0140 -6736(08)61081-8.

25. Nachega JB, Parienti JJ, Uthman OA, Gross R, Dowdy DW, Sax PE, et al. Lower pill burden and once-daily antiretroviral treatment regimens for HIV infection: a meta-analysis of randomized controlled trials. Clin Infect Dis. 2014;58:1297-307. https://doi.org/10.1093/ cid/ciu046.
26. Stecher M, Schommers P, Schmidt D, Kollan C, Gunsenheimer-Bartmeyer B, Lehmann C, et al. Antiretroviral treatment indications and adherence to the German-Austrian treatment initiation guidelines in the German ClinSurv HIV Cohort between 1999 and 2016. Infection. 2019;47:247-55. https://doi.org/10.1007/s15010-018-1248-8.

27. Penafiel J, de Lazzari E, Padilla M, Rojas J, Gonzalez-Cordon A, Blanco JL, et al. Tolerability of integrase inhibitors in a real-life setting. J Antimicrob Chemother. 2017;72:1752-9. https://doi. org/10.1093/jac/dkx053.

28. Greenberg L, Ryom L, Wandeler G, Grabmeier-Pfistershammer K, Öllinger A, Neesgaard B, et al. Uptake and discontinuation of integrase inhibitors (INSTIs) in a large cohort setting. J Acquir Immune Defic Syndr. 1999;2020(83):240-50. https://doi.org/10.1097/ qai.0000000000002250

29. Bourgi K, Rebeiro PF, Turner M, Castilho JL, Hulgan T, Raffanti $\mathrm{SP}$, et al. Greater weight gain in treatment naive persons starting dolutegravir-based antiretroviral therapy. Clin Infect Dis. 2019. https ://doi.org/10.1093/cid/ciz407.

30. Hoffmann C, Welz T, Sabranski M, Kolb M, Wolf E, Stellbrink $\mathrm{HJ}$, et al. Higher rates of neuropsychiatric adverse events leading to dolutegravir discontinuation in women and older patients. HIV Med. 2017;18:56-63. https://doi.org/10.1111/hiv.12468.

\section{Affiliations}

\section{Melanie Stecher ${ }^{1,2} \odot \cdot$ Philipp Schommers ${ }^{1,2} \cdot$ Christian Kollan $^{3} \cdot$ Matthias Stoll $^{4} \cdot$ Frieder Kuhlendahl $^{5}$. Hans-Jürgen Stellbrink ${ }^{6}$. Jan-Christian Wasmuth ${ }^{7,8}$. Christoph Stephan ${ }^{9} \cdot$ Laura Hamacher $^{1}$. Clara Lehmann ${ }^{1,2}$. Christoph Boesecke ${ }^{7}$. Johannes Bogner ${ }^{10}$. Stefan Esser ${ }^{11}$. Carlos Fritzsche ${ }^{12} \cdot$ Annette Haberl $^{9}$. Dirk Schürmann ${ }^{16}$. Olaf Degen ${ }^{18}$. Heinz-August Horst ${ }^{19}$. Christian Hoffmann ${ }^{13}$. Björn Jensen ${ }^{14}$. Carolynne Schwarze-Zander ${ }^{7}$. Martin Platten $^{15}$. Gerd Fätkenheuer ${ }^{1,2}$. Daniel Schmidt ${ }^{3,16}$. Barbara Gunsenheimer-Bartmeyer ${ }^{3}$. Jörg Janne Vehreschild ${ }^{1,2,17}$. On behalf of the ClinSurv Study Group}

1 Department I for Internal Medicine, Faculty of Medicine and University Hospital Cologne, University of Cologne, Herderstraße 52-54, 50931 Cologne, Germany

2 German Center for Infection Research (DZIF), Partner Site Bonn-Cologne, Cologne, Germany

3 Robert-Koch Institute (RKI), Berlin, Germany

4 Department of Clinical Immunology and Rheumatology, Hannover Medical School, Hannover, Germany

5 Ifi-Institute for Interdisciplinary Medicine, Hamburg, Germany

6 Infectious Disease Medical Center, Hamburg, Germany

7 Department I for Internal Medicine, University Hospital of Bonn, Bonn, Germany

8 German Center for Infection Research (DZIF), Partner Site Bonn-Cologne, Bonn, Germany

9 Department of Infectious Diseases, University Hospital Frankfurt, Goethe-University, Frankfurt am Main, Germany

10 Section for Infectious Diseases, Medical Clinic and Polyclinic IV, University Hospital, Ludwig-Maximilians-University Munich, Munich, Germany
11 Clinic of Dermatology, Department of Venerology, University Hospital Essen, Essen, Germany

12 Department of Tropical Medicine and Infectious Diseases, University of Rostock, Rostock, Germany

13 ICH Study Center, Infektionsmedizinisches Centrum Hamburg, Hamburg, Germany

14 Department of Gastroenterology, Hepatology and Infectiology, University of Düsseldorf, Düsseldorf, Germany

15 Labor Dr. Wisplinghoff, Cologne, Germany

16 Charité - University Medicine Berlin, Berlin, Germany

17 Department of Hematology and Oncology, Johann Wolfgang Goethe University of Frankfurt, Frankfurt am Main, Germany

18 University Clinic Hamburg Eppendorf, Hamburg, Germany

19 University Hospital Schleswig-Holstein, Kiel, Germany 